\title{
INTER-RATER AND INTRA-RATER RELIABILIY OF POSTURAL ANALYSIS SOFTWARE(MATLAB 7.11 R2010B)
}

\author{
Bindesh D. Patel ${ }^{* 1}$,Payal B. Rathi ${ }^{2}$, Nirav N. Viradiya ${ }^{3}$. \\ ${ }^{*}$ Deputy Registrar, P P Savani University, Mangrol, Surat, Gujarat, India. \\ ${ }^{2}$ Pediatric Physiotherapist, Foster Physio \& Sports, Surat, Gujarat, India. \\ ${ }^{3}$ Clinical Physiotherapist, Foster Physio \& Sports, Surat, Gujarat, India.
}

\section{ABSTRACT}

\begin{abstract}
Introduction:Posture analysis software Matlab 7.11 R2010b is commonly used as a tool for postural assessment. It is easy to use. Nonetheless, reliability studies are lacking.

Methods: The study sample consisted of 25 pictures from 25 subjects, and each subject was assessed ( 1 day interval) by 3 blinded raters and by 1 blind rater for 3 times. Photographs of subject were coded and two variables (Lumbar lordosis and Pelvic tilt) were analyzed. Inter- and intra-rater reliabilities were calculated using intra-class correlation coefficient.

Results: The intra-class correlation coefficient (ICC) was calculated. We found excellent inter-rater reliability and intra-rater reliability of Posture analysis software Matlab7.11 R2010b for both variables.

Discussion: Observation posture analysis is easy but it is less reliable method. It cannot be used to measure progression of posture corrections and for research purpose. Photography method is most reliable method to evaluate posture. There are many softwares available for analysis of posture and Matlab 7.11 R2010b is one of them. In this study we found excellent inter-rater reliability and intra-rater reliability of the software.

Conclusion:The postural assessment software Matlab 7.11 R2010b is having excellent inter-rater reliability and intra-rater reliability and should be considered as a reliable tool for postural assessment.

KEYWORDS: Matlab, Posture, Reliability, Lumbar lordosis, Pelvic Tilt.
\end{abstract}

Address for correspondence: Dr. Bindesh D. Patel, Deputy Registrar, P P Savani University, Mangrol, Surat, Gujarat, India. Mobile: 9998667396, E-Mail:bindesh.hpe@gmail.com
Access this Article online
Journal Information
Quick Response code

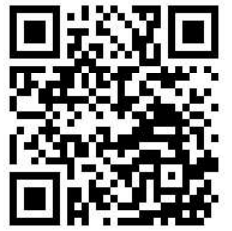
DOI: $10.16965 /$ ijpr.2020.124
International Journal of Physiotherapy and Research
ISSN (E) 2321-1822 | ISSN (P) 2321-8975
https://www.ijmhr.org/ijpr.html
DOI-Prefix: https://dx.doi.org/10.16965/ijpr
Article Information
Received: 28 Mar 2020
Peer Review: 28 Mar 2020
Revised: None
Accepted: 15 May 2020
Published (O): 11 Jun 2020
Published (P): 11 Jun 2020

(c) $\mathrm{ET}-\mathrm{NB}-\mathrm{SA}$

\section{INTRODUCTION}

Posture has been defined as the alignment of body segments at a given point of time. Posture is considered as an important marker of health and postural abnormalities are associated with a large number of disorders, including pain syndromes, generalized or regional musculoskeletal disorders, and respiratory dysfunctions [1].

Assessment of posture is an important component of the evaluation and affects the design of a treatment regimen [2].Quantitative measurements allow physicians and researchers not only to make an accurate assessment of postural changes but also to monitor improvement [1].Correct upright posture is considered to be an important indicator of musculoskeletal health [3].

The main reasons for carrying out postural assessment are to acquire information, save time, establish a baseline and to treat holistically. Analysis of posture as part of assessment offers a complete service in keeping with the 
idea of treating people holistically and not compartmentalized [4].

A variety of postural assessment methods have been in use. Some are conventional, while some are latest and few are those which got modified into latest form from conventional forms i.e. these evolved into better and convenient methods. Visual observation is the commonest method which is used to assess posture in clinical practice. The one and only advantage of this method is that it does not require any equipment. With this method, quantitative data cannot be obtained. Thus, minor postural alterations cannot be detected. Also, it has been reported to have a poor interrater agreement [5]. Photography or filming methods enable clinicians to detect postural changes with time, and inter-relate various body parts through measurements with specific software. Photography or filming are low cost methods which are easy and quick to use, but they require several methodological steps to standardize the photos/ films and prevent or reduce distortions and measurement errors. Moreover, there are some limitations inherent to the instruments, such as the analytical variations resulting from the choice of an image that does not correspond exactly to the anatomic plane under evaluation, which is called parallax. Since photography provides a two dimensional image, only the part of the body that is in focus has true measurements, while the other parts might have their dimensions distorted by the effect of parallax. Software is currently available to process two dimensional images, captured simultaneously by several cameras, and reconstruct them in 3D for analysis [4].

MATLAB is a multi-paradigm numerical computing environment and proprietary programming language developed by MathWorks. It allows the measurement of angles. The software is easy to use, and it is accompanied by scientific tutorials. We envision that MATLAB 7.11 R2010B can be broadly used in both clinical practice and research if excellent reliability is found.

The present study evaluated inter-rater (repeatability) and intra-rater (reproducibility) reliabilities of MATLAB 7.11 R2010B for assessing posture angles. We hypothesized that MATLAB 7.11 R2010B would be an accurate tool for postural assessments.

\section{METHOD}

The study sample consisted of 25 subjects, age varies between 30 to 50 years, participated in the study (gender $=$ Male; age $=39.92$ years). None of the participants had injuries or diseases of the musculoskeletal or nervous system or had been taking medications that would affect standing posture six months prior to participation. All research was done at MGM School of Physiotherapy. Participants provided informed consent.

Data collection: In total, 25 pictures were taken in the side view. The sample size and number of pictures were chosen based on the relevant literature. Pictures were taken with subjects in the standing position, and the subjects were dressed with swimming suit so that body parts were properly exposed. Markers were attached at C3, C7, L4, ASIS, PSIS and sternal notch with the help of double sided adhesive tape. Small sized blue balls were used to mark the bony landmarks, with the help of double sided adhesive tape.Participants were asked to stand on foot print drawn over mat. They were instructed to stand as steady \& symmetrically as possible. White curtain was fixed with the help of adhesive tape against wall for clear background in photographs. Mat was used with foot print drawn with $8.5 \mathrm{~cm}$ heels apart and toes are pointing outward at $9^{\circ}$ from sagittal plane. Sony cyber shot camera with 12.1 megapixels was used. Camera was aligned vertically for full view of the body. Camera was positioned on camera stand 43 inch from ground and camera stand was placed 90 inch away from wall. Zooming was not done. All photos were taken by same Physiotherapist. Subjects were asked to stand on footprint drawn over mat and then photographs were taken from right side for right sagittal view. Three photographs were taken of each subject and best one was selected. These photographs were transferred from camera to computer in which MATLAB software was installed. All photographs were coded and analysed with the help of software for various angles. Lumbar lordosis, pelvic tilt, trunk lean angle and neck angle were measured using posture evaluation software by MATLAB 7.11 R2010B. Out of all angles randomly two variables (Lumbar lordosis and Pelvic tilt) were 
included in the study [6].

Inter-rater reliability data analysis procedure: Three raters, identified here as rater 1,2 and 3 were used for inter-rater reliability evaluation. All raters were certified Physiotherapist. All rater were given the coded 25 photographs of 25 subjects. Each rater was blinded with the result of other raters. All rater were not the regular user of the software. Raters were oriented about how to use the software and they practiced by analyzing 8 pictures other than coded 25 photographs of 25 subjects. Each rater had 30 minutes to practice and could clear their doubts during the practice time. Then the all the coded photographs were given to the raters and were asked them to do the analysis on different days.

Intra-rater reliability data analysis procedure: Only rater 1 was used for the intra-rater reliability trial. He was also given the training same as the inter-rater method and this rater tested all 25 photographs on 3 separate occasions within 48 hours period, using the same assessment technique.

Fig. 1: Items used in study.

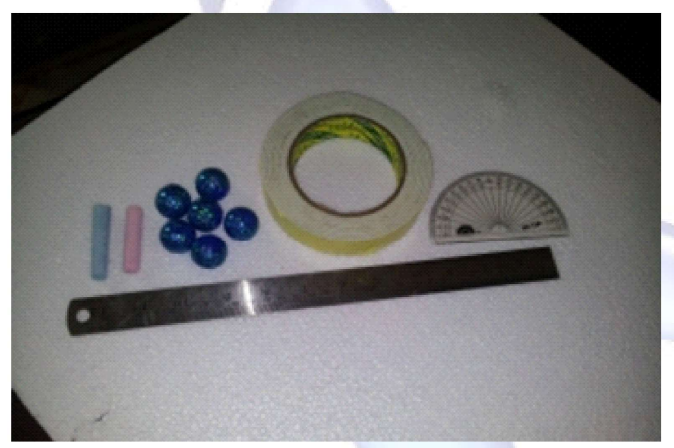

Details: Figure is showing the scale, small blue markers, semi-circle, double sided adhesive tape and chalk stick. Scale, chalk stick and semi-circle was used to draw foot print. Double sided adhesive tape was used to stick the small blue markers on the body of subject.

Fig. 2:Camera

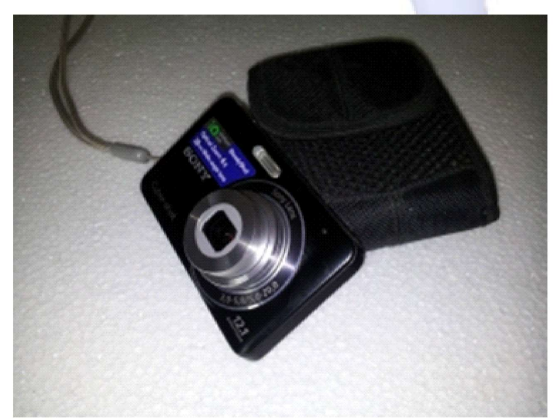

Details: - This figure shows the camera being used in this study.
Fig. 3:Foot print.

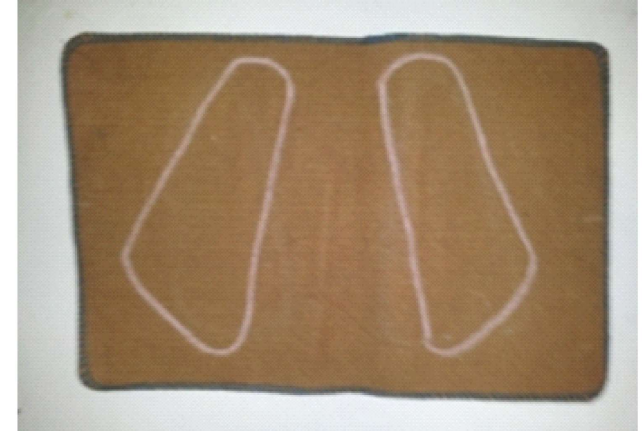

Details: - This figure shows the footprint on which subject was supposed to stand for the uniform foot position.

Fig. 4:Lumbar lordosis assessment.

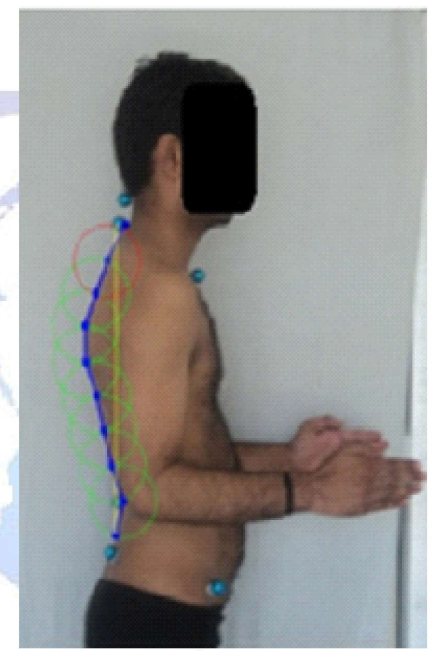

Details: - This figure is a screen shot of the software which shows the measurement technique of the spinal curve which includes thoracic kyphosis and lumbar lordosis.

Fig. 5:Pelvic tilt assessment.

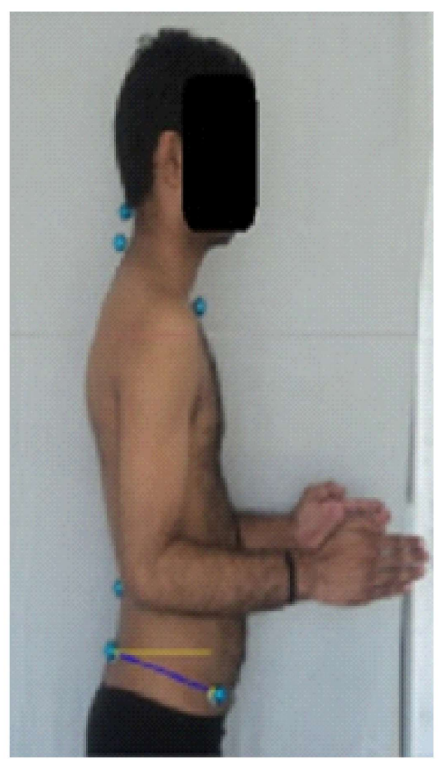

Details: - This figure is also a screen shot of the software which shows the pelvic tilt angle measurement technique. 


\section{RESULT}

Analyses were conducted using SPSS software. The intra-class correlation coefficient (ICC) model 2.130 was employed for the inter-rater tests and ICC 3.130 was employed for the intrarater tests. The significance level was defined as $\alpha=0.05$.

Inter-rater reliability (reproducibility): Table 1 displays the inter-rater reliability and mean of both the variables. The ICC guideline is given by Koo and Li (2016) 20

- below 0.50: poor

- between 0.50 and 0.75 : moderate

- between 0.75 and 0.90: good

- above 0.90: excellent

Both variables have excellent inter-rater reliability.

Table 1: ICC of both variables for inter-rater reliability.

\begin{tabular}{|c|c|c|c|c|c|}
\hline Sr. No & Variable & Abbreviation & ICC & $95 \% \mathrm{Cl}$ & Mean \\
\hline 1 & Lumbar Lordosis & LL & $.916^{\mathrm{C}}$ & $.836-.960$ & \\
\hline 2 & Pelvic tilt & PT & $.987^{\mathrm{C}}$ & $.976-994$ & \\
\hline
\end{tabular}

Graph 1 Linear presentation of lumbar lordosis variable for inter-rater reliability.

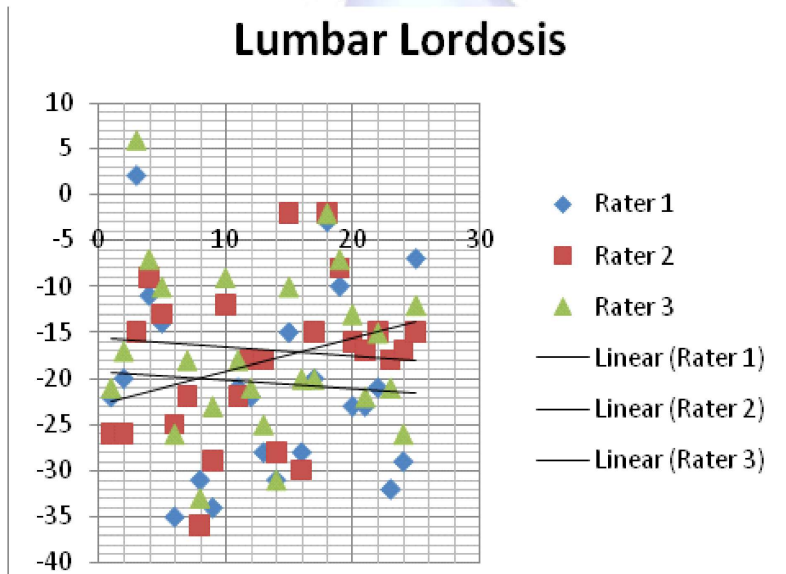

Graph 2: Linear presentation of Pelvic tilt variable for inter-rater reliability.

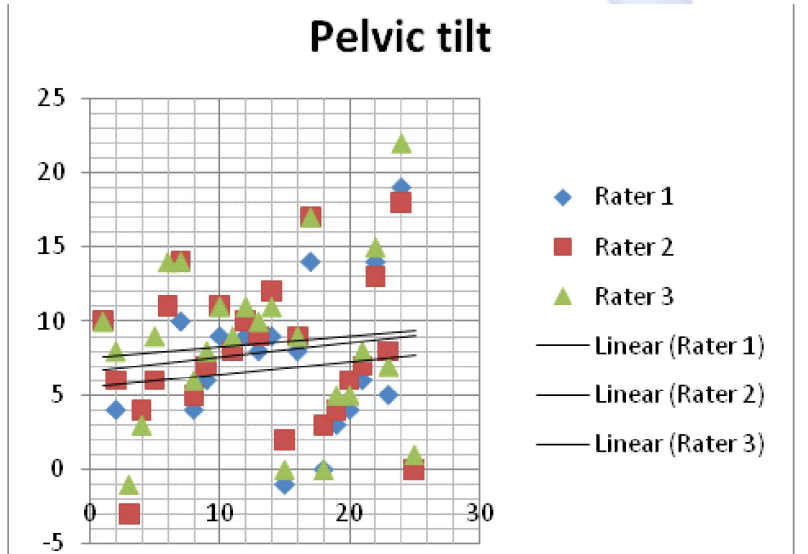

Intra-rater reliability (repeatability): Table 2 displays the intra-rater reliability and mean. The ICC guideline is given by Koo and Li (2016) 20 - below 0.50: poor

- between 0.50 and 0.75: moderate

- between 0.75 and 0.90: good

- above 0.90: excellent

Both variables have excellent intra-rater reliability.

Table 2: ICC of both variables for intra-rater reliability.

\begin{tabular}{|c|c|c|c|c|c|}
\hline Sr. No & Variable & Abbreviation & ICC & $95 \% \mathrm{Cl}$ & Mean \\
\hline 1 & Lumbar Lordosis & LL & $.953^{\mathrm{C}}$ & $.909-.978$ & \\
\hline 2 & Pelvic tilt & PT & $.988^{\mathrm{C}}$ & $.977-.994$ & \\
\hline
\end{tabular}

Graph 3: Linear presentation of lumbar lordosis variable for intra-rater reliability.

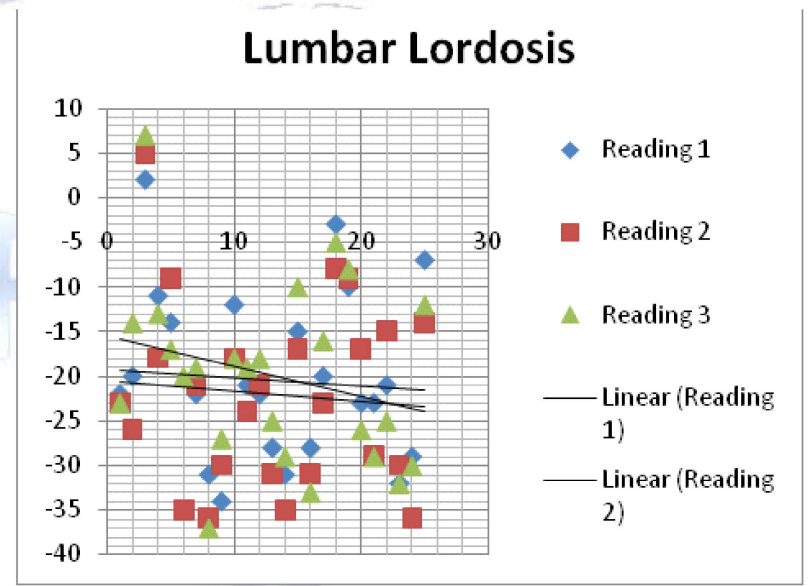

Graph 4: Linear presentation of Pelvic tilt variable for intra-rater reliability.

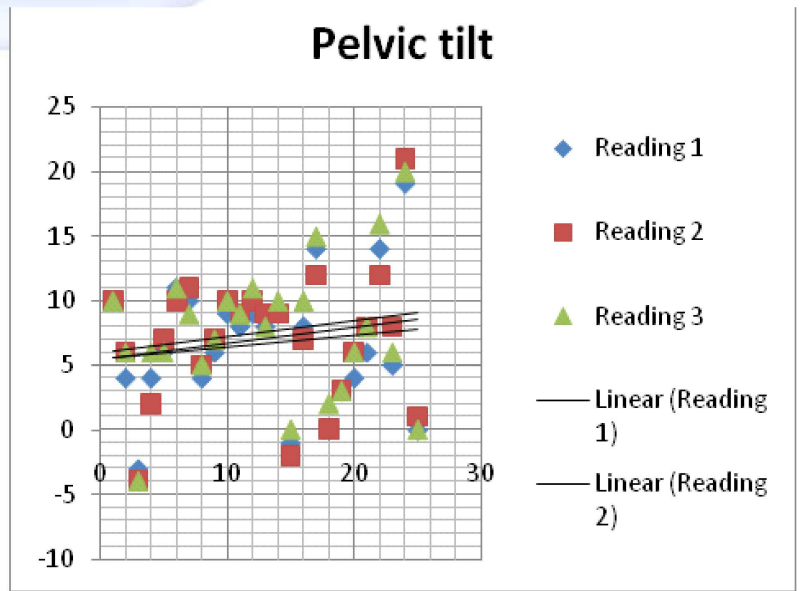

DISCUSSION

The present study measured the inter-and intra-rater reliabilities of the MATLAB 7.11 R2010B. We found that the MATLAB R2010B is a reliable tool for postural analysis because intra-class correlation coefficient (ICC) for inter-rater reliability and intra-rater reliability is excellent. 
Evaluation of body posture, using well defined anatomical landmarks in their respective relation to a central plumb line, as was reported by Kendall and McCreary, is subjective as well. A difference in interpretation can occur. Although body posture photography takes more time in patient education and production than the usual inspection in the daily routine physical therapy practice, the photographic procedure has some obvious advantages:

- The results of the photography seem more objective than the description of the an amnestic inspection in daily practice. A deviation of postural alignment seems easier detectable on a photograph. Accuracy of both Kendall's and Griegel-Morris' methods for evaluation of posture are affected by their estimated measures.

- The collected data of the various postural aspects of the subjects can be recorded in a computerized database.

- It is a non-invasive procedure for the subjects that need to be screened with the method.

- Standardization of the photographic procedure and the measurement procedure will provide repeatable and reliable data [7].

Stephen C. Cobb and Roger James investigated the reliability and validity of digital photographic measurement method (DPMM) technology. They found DPMM technology valid and reliable clinical and research tool. In their study they have investigated the reliability of the method and they have mentioned that reliability of method also depends on the individual who is marking the bony landmark and to eliminate that factor in our study palpation and photographs were taken by one individual only. And same photographs were given to the different raters for the assessment [8].

Elizabeth and Marcos Duarte also did the study of validity and reliability of Posture assessment software and they found software accurate for measuring corporal angles and distances mentioned that it should be con-sidered as a reliable tool for postural assessment. Detail of software was not mentioned in the study [1].

Barbara Luflerty and Louis R. Amunds on did the quantitative assessment of head and shoulder posture and found the Posture Analysis Digitizing System (PADS) a reliable method for the assessment of head and shoulder posture [2].

Carole Fortin and associates did study on reliability of a quantitative clinical posture assessment tool among persons with idiopathic scoliosis and concluded that photographic method is reliable method to assess the posture and future research can be done to find the suitable tool to measure the progression of posture correction[9].

\section{CONCLUSION}

From the above research we can conclude that MATLAB 7.11 R2010B is the reliable software to evaluate the posture and can be used in clinical and research set up.

\section{ACKNOWLEDGEMENTS}

I would like to thank Dr. Rajani Mullerpatan for her continuous support and motivation. I am also thankful to Dr. Yuvraj Singh for continuous guidance during the research.

\section{Conflicts of interest: None}

\section{REFERENCES}

[1]. Elizabeth Alves G. Ferreira et al. POSTURAL ASSESSMENT SOFTWARE (PAS/SAPO): VALIDATION AND RELIABILIY.CLINICS 2010;65(7):675-81 doi: 10.1590/ S1807-59322010000700005

[2]. Barbara Luflerty Braun et al. Quantitative assessment of head and shoulder posture. Arch Phys Med Rehabil1989;70:322-329.

[3]. Maureen P McEvoy et al. Reliability of upright posture measurements in primary school children. BMC Musculoskeletal Disorders 2005;6:35. doi:10.1186/ 1471-2474-6-35

[4]. Purva C. Badhe et al. A Review on Posture Assessment. IOSR Journal of Sports and Physical Education (IOSR-JSPE) 2018;5(5):08-15.

[5]. Deepika Singla et al. Methods of Postural Assessment Used for Sports Persons. Journal of Clinical and Diagnostic Research. 2014 Apr;8(4):LE01-LE04.

[6]. ACH Geurts,TABoonstra,NCVoermans, MGDiender, VWeerdesteyn, BRBloem.Assesment of postural asymmetric in mild to moderate parkinson's disease. Gait Posture 2011;33:143-145.

[7]. Florence Peterson Kendall,Elizabeth Kendall McCreary,PatriciaGeiseProvance.Muscles testing and function. 4th edition;1993

[8]. Stephen Cet al.A Digital Photographic Measurement Method for Quantifying Foot Posture: Validity, Reliability, and Descriptive Data. Journal of Athletic Training 2011;46(1):20-30

[9]. Carole Fortin et al. Reliability of a quantitative clinical posture assessment tool among persons with idiopathic scoliosis. Physiotherapy2012;98:64-75. 